\title{
On Effects of a New Method for Fractional Initial Value Problems
}

\author{
Hülya Kodal Sevindir $\mathbb{D}$, Süleyman Çetinkaya $\mathbb{D}$, and Ali Demir
}

Department of Mathematics, University of Kocaeli, Kocaeli 41380, Turkey

Correspondence should be addressed to Hülya Kodal Sevindir; hkodal@kocaeli.edu.tr

Received 21 September 2021; Revised 1 November 2021; Accepted 25 November 2021; Published 24 December 2021

Academic Editor: Zengtao Chen

Copyright (C) 2021 Hülya Kodal Sevindir et al. This is an open access article distributed under the Creative Commons Attribution License, which permits unrestricted use, distribution, and reproduction in any medium, provided the original work is properly cited.

\begin{abstract}
The aim of this study is to analyze nonlinear Liouville-Caputo time-fractional problems by a new technique which is a combination of the iterative and ARA transform methods and is denoted by IAM. First, the ARA transform method and its inverse are utilized to get rid of time fractional derivative. Later, the iterative method is applied to establish the solution of the problem in infinite series form. The main advantages of this method are that it converges to analytic solution of the problem rapidly and implementation of method is easy. Finally, outcomes of the illustrative examples prove the efficiency and accuracy of the method.
\end{abstract}

\section{Introduction}

The mathematical problems including fractional differential equations play a significant role in analysis and modelling of various scientific processes such as damping laws, electrical circuits, fluid mechanics, and relaxation processes since fractional derivative is nonlocal operator [1-5]. As a result, fractional mathematical problems attract a growing attention of numerous scientist from diverse branches of science. The adversity of fractional differential equations is that solving them analytically is hard or impossible. Therefore, numerous numerical methods such as reduced differential transform method (RDTM) [6], Adomian decomposition method (ADM) [7], homotopy perturbation method (HPM) [8], variational iteration method (VIM) [9, 10], homotopy analysis method (HAM) [11], fractional difference method (FDM) [12], and new iterative method (NIM) [13] have been developed to establish numerical solution in series form.

ARA transform is a new integral transform method to tackle with any kind of differential equations. The differential equation is reduced into algebraic equation or simpler differential equation by this method. Moreover, the ARA transform method is more applicable than the Laplace transform method since the domain of this method covers the domain of the Laplace transform method [14]. Combination of this transform with other numerical methods produces new and effective methods to construct numerical or analytical solution of differential equations. In this study, the combination of ARA transform method with iterative method is utilized to construct the solution of nonlinear fractional differential problems of biological population. Iterative method is a very common method to obtain the numerical solution of mathematical problems including differential equations [15].

The main goal of this research is to establish numerical or analytical solutions of nonlinear Liouville-Caputo timefractional problems by means of IAM which is a new effective and versatile method. The novelty of this study is that this is the first study in which ARA transform method is applied to fractional differential problem including fractional equation in Liouville-Caputo sense.

The rest of the paper is organized as follows: fundamental definitions and properties of fractional calculus and ARA transform, the ARA transform of fundamental functions, and existence of ARA transform for Liouville-Caputo derivative and Riemann-Liouville integral are given in Section 2. Implementation and convergence analysis of the method for nonlinear fractional mathematical problems is presented in Section 3. Illustrative examples including LiouvilleCaputo time-fractional biological population problem are 
presented and analyzed in Section 4. Finally, the outcomes of this method are presented in conclusion.

\section{Preliminary Results}

In this section, preliminaries, notations, and features of the fractional calculus are given $[12,16]$.

Definition 1. Riemann-Liouville time-fractional integral of a real valued function $u(t)$ is defined as

$$
I_{t}^{\alpha} u(t)=\frac{1}{\Gamma(\alpha)} \int_{0}^{t}(t-s)^{\alpha-1} u(s) d s
$$

where $\alpha>0$ denotes the order of the integral.

Definition 2. $\alpha^{\text {th }}$ order of the Liouville-Caputo fractional derivative of $u(t)$ is defined as

$$
D_{t}^{\alpha} u(t)=\frac{1}{\Gamma(n-\alpha)} \int_{t_{0}}^{t}(t-s)^{n-\alpha-1} u^{(n)}(s) d s, t \in\left[t_{0}, t_{0}+T\right]
$$

where $n-1<\alpha<n$ and $u^{(n)}(t)=d^{n} u / d t^{n}$. If $\alpha$ is an integer, then the Liouville-Caputo fractional derivative becomes the integer-order derivative.

Definition 3. $\alpha^{\text {th }}$ order of the Liouville-Caputo fractional derivative of $u(t)$ is defined as

$$
D_{t}^{\alpha} u(t)=\frac{1}{\Gamma(1-\alpha)} \int_{t_{0}}^{t}(t-s)^{-\alpha} u^{\prime}(s) d s, t \in\left[t_{0}, t_{0}+T\right]
$$

where $0<\alpha<1$.

Definition 4. The Mittag-Leffler function with the parameters $\alpha$ and $\beta$ is given as follows [17].

$$
E_{\alpha, \beta}\left(\lambda\left(t-t_{0}\right)^{\alpha}\right)=\sum_{k=0}^{\infty} \frac{\left(\lambda\left(t-t_{0}\right)^{\alpha}\right)^{k}}{\Gamma(\alpha k+\beta)}, \alpha, \beta>0, \lambda \in R
$$

If $t_{0}=0, \alpha=\beta=\mathrm{q}$, then we get

$$
E_{q, \mathrm{q}}\left(\lambda t^{q}\right)=\sum_{k=0}^{\infty} \frac{\left(\lambda t^{q}\right)^{k}}{\Gamma(\alpha k+\beta)}, q>0
$$

Moreover, substituting $q=1$, in equation (5), we have $E_{1,1}(\lambda t)=e^{\lambda t}$. If the reader wants more information, they should refer to [17]. The following functions are used to obtain the solution of the problem discussed in this study.

Definition 5. The ARA integral transform of order $n$ of the continuous function $g(t)$ on the interval $(0, \infty)$ is defined as [14]

$$
G_{n}[g(t)](s)=G(n, s)=s \int_{0}^{\infty} t^{n-1} e^{-s t} g(t) d t, s>0
$$

Definition 6. The inverse of the ARA transform is given by

$$
\begin{aligned}
g(t)= & G_{n+1}^{-1}\left[G_{n+1}[g(t)]\right] \\
= & \frac{(-1)^{n}}{2 \pi i} \int_{c-i \infty}^{c+i \infty} e^{s t}\left(( - 1 ) ^ { n } \left(\frac{1}{s \Gamma(n-1)}\right.\right. \\
& \left.\left.\cdot \int_{0}^{s}(s-x)^{n-1} G(n+1, x) d x+\sum_{k=0}^{n-1} \frac{s^{k}}{k !} \frac{\partial^{k} G(0)}{\partial s^{k}}\right)\right) d s,
\end{aligned}
$$

where $G(s)=\int_{0}^{\infty} e^{-s t} g(t) d t$ is $(n-1)$ times differentiable [14].

Now, significant properties which play a vital role for the solution of fractional differential equations are presented as follows:

Property 7. The ARA transform of Mittag-Leffler for $n=1$ are computed as follows:

$$
\begin{aligned}
G_{n} & {\left[t^{\beta-1} E_{\alpha, \beta}\left(\lambda t^{\alpha}\right)\right](s) } \\
& =s \int_{0}^{\infty} t^{n-1} e^{-s t} t^{\beta-1} \sum_{k}^{\infty}\left(\left(\lambda t^{\alpha}\right)^{k} / \Gamma(\alpha k+\beta)\right) d t \\
& =\sum_{k}^{\infty} \frac{\lambda^{k}}{\Gamma(\alpha k+\beta)} s \int_{0}^{\infty} e^{-s t} t^{n+\beta-2+\alpha k} d t \\
& =\sum_{k}^{\infty} \frac{\lambda^{k}}{\Gamma(\alpha k+\beta)} s \int_{0}^{\infty} t^{n-1} e^{-s t} t^{\beta-1+\alpha k} d t \\
& =\sum_{k}^{\infty} \frac{\lambda^{k}}{\Gamma(\alpha k+\beta)} \frac{\Gamma(\beta-1+\alpha k+n)}{s^{\beta-1+\alpha k+n-1}} \\
& =\frac{1}{s^{\beta+n-2}} \sum_{k}^{\infty} \frac{\lambda^{k}}{\Gamma(\alpha k+\beta)} \frac{\Gamma(\beta+\alpha k+n-1)}{s^{\alpha k}} .
\end{aligned}
$$

For $n=1$,

$$
\begin{aligned}
G_{1} & {\left[t^{\beta-1} E_{\alpha, \beta}\left(\lambda t^{\alpha}\right)\right](s) } \\
& =\frac{1}{s^{\beta-1}} \sum_{k}^{\infty} \frac{\lambda^{k}}{\Gamma(\alpha k+\beta)} \frac{\Gamma(\beta+\alpha k)}{s^{\alpha k}} \\
& =\frac{1}{s^{\beta-1}}\left(\frac{1}{1-\left(\lambda / s^{\alpha}\right)}\right)=\frac{s^{\alpha-\beta+1}}{s^{\alpha}-\lambda} .
\end{aligned}
$$

Property 8. The ARA transform of $t^{p \alpha}$ for $p \in \mathbb{N}$ is defined as follows: 


$$
\begin{aligned}
G_{n}\left[t^{p \alpha}\right](s) & =s \int_{0}^{\infty} t^{n-1} e^{-s t} t^{p \alpha} d t=s \int_{0}^{\infty} t^{p \alpha+n-1} e^{-s t} d t \\
& =\Gamma(p \alpha+n)\left(\frac{1}{s}\right)^{p \alpha+n} s \int_{0}^{\infty} \frac{t^{p \alpha+n-1} e^{-s t}}{\Gamma(p \alpha+n)(1 / s)^{p \alpha+n}} d t \\
& =\Gamma(p \alpha+n)\left(\frac{1}{s}\right)^{p \alpha+n} s=\frac{\Gamma(p \alpha+n)}{s^{p \alpha+n-1}} .
\end{aligned}
$$

Property 9 [14]. The ARA transform of convolution is defined as follows:

$$
G_{n}[f(t) * g(t)](s)=(-1)^{n-1} s \sum_{j=0}^{n-1} c_{j}^{n-1} F^{(j)}(s) G^{(n-1-j)}(s) .
$$

For $n=1$, it becomes

$$
G_{1}[f(t) * g(t)](s)=s F(s) G(s),
$$

where $G(s)=\int_{0}^{\infty} e^{-s t} g(t) d t$ and $F(s)=\int_{0}^{\infty} e^{-s t} f(t) d t$.

Theorem 10 (the existence of ARA transform for RiemannLiouville integral). If the Riemann-Liouville integral ${ }_{0}^{R L} I_{t}^{\alpha} f(t)$ of the function $f(t)$ is piecewise continuous in every finite interval and fulfils

$$
\mid t^{n-1}\left\{_{0} R L I\right\}_{t^{\alpha f}}(t) \leq K e^{\beta t},
$$

then ARA transform exists for all $s>\beta$.

Proof. By the property of integration the ARA transform of the Riemann-Liouville integral leads to the following

$$
\begin{aligned}
s \int_{0}^{\infty} t^{n-1} e_{0}^{-s t} R L I_{t}^{\alpha} f(t) d t= & s \int_{0}^{\gamma} t^{n-1} e^{-s t}{ }_{0}^{R L} I_{t}^{\alpha} f(t) d t \\
& +s \int_{\gamma}^{\infty} t^{n-1} e_{0}^{-s t}{ }_{0} L I_{t}^{\alpha} f(t) d t
\end{aligned}
$$

piecewise continuity of ${ }_{0}^{R L} I_{t}^{\alpha} f(t)$ implies the existence of first integral on the right side, and the convergence of improper integral on the right side is shown below:

$$
\begin{aligned}
\left|s \int_{\gamma}^{\infty} t^{n-1} e^{-s t}{ }_{0}^{R L} I_{t}^{\alpha} f(t) d t\right| \leq & s \int_{\gamma}^{\infty} e^{-s t}\left|t_{0}^{n-1}{ }_{0}^{R L} I_{t}^{\alpha} f(t)\right| d t \\
\leq & s \int_{\gamma}^{\infty} K e^{\beta t-s t} d t=\lim _{c \longrightarrow \infty} \\
& -\left.s K \frac{e^{-t(s-\beta)}}{s-\beta}\right|_{\gamma} ^{c}=\frac{s K}{s-\beta} e^{-\gamma(s-\beta)} .
\end{aligned}
$$

As a result, the ARA transform of ${ }_{0}^{R L} I_{t}^{\alpha} f(t)$ exists
Theorem 11 (ARA transform of Riemann-Liouville integral for $n=1)$. If the Riemann-Liouville integral ${ }_{0}^{R L} I_{t}^{\alpha} f(t)$ of the function $f(t)$ is piecewise continuous in every finite interval and fulfils

$$
\left|t^{n-1} \underset{0}{R L} I_{t}^{\alpha} f(t)\right| \leq K e^{\beta t}
$$

then ARA transform of it for $n=1$ is computed as

$$
G_{1}\left[\begin{array}{l}
R L \\
0
\end{array} I_{t}^{\alpha} f(t)\right](s)=\frac{1}{s^{\alpha}} G_{1}[f(t)](s),
$$

for all $s>\beta$.

Proof. For $n=1$, we have

$$
G_{1}\left[{ }_{0}^{R L} I_{t}^{\alpha} f(t)\right](s)=G_{1}\left[\frac{1}{\Gamma(\alpha)} \int_{0}^{t}(t-x)^{\alpha-1} f(x) d x\right](s) .
$$

In terms of convolution, it can be rewritten as follows

$$
G_{1}\left[\frac{1}{\Gamma(\alpha)}\left[t^{\alpha-1} * f(t)\right]\right](s)
$$

by using convolution property of the ARA transform, we reach the following

$$
\begin{aligned}
& \frac{1}{\Gamma(\alpha)} s\left(\int_{0}^{\infty} e^{-s t} t^{\alpha-1} d t\right)\left(\int_{0}^{\infty} e^{-s t} f(t) d t\right) \\
& \quad=\frac{1}{\Gamma(\alpha)} s \frac{\Gamma(\alpha)}{s^{\alpha}}\left(\int_{0}^{\infty} e^{-s t} f(t) d t\right) \\
& \quad=\frac{1}{s^{\alpha}} s \int_{0}^{\infty} e^{-s t} f(t) d t=\frac{1}{s^{\alpha}} G_{1}[f(t)](s) .
\end{aligned}
$$

Theorem 12 (the existence of ARA transform for LiouvilleCaputo derivative). If the $\alpha^{\text {th }}$ order of Liouville-Caputo derivative ${ }_{0}^{C} D_{t}^{\alpha} f(t)$ of the function $f(t)$ is piecewise continuous in every finite interval, $f(t)$ is $n$ times continuously differentiable and fulfils

$$
\left|t^{n-1}{ }_{0}^{C} D_{t}^{\alpha} f(t)\right| \leq K e^{\beta t}
$$

then ARA transform exists for all $s>\beta$.

Proof. By the property of integration the ARA transform of the Liouville-Caputo derivative leads to the following

$$
\begin{aligned}
& s \int_{0}^{\infty} t^{n-1} e^{-s t}{ }_{0}^{C} D_{t}^{\alpha} f(t) d t \\
& \quad=s \int_{0}^{\gamma} t^{n-1} e^{-s t}{ }_{0}^{C} D_{t}^{\alpha} f(t) d t+s \int_{\gamma}^{\infty} t^{n-1} e^{-s t}{ }_{0}^{C} D_{t}^{\alpha} f(t) d t,
\end{aligned}
$$


piecewise continuity of ${ }_{0}^{C} D_{t}^{\alpha} f(t)$ implies the existence of first integral on the right side, and the convergence of improper integral on the right side is shown below:

$$
\begin{aligned}
& \left|s \int_{\gamma}^{\infty} t^{n-1} e^{-s t}{ }_{0}^{C} D_{t}^{\alpha} f(t) d t\right| \\
& \quad \leq s \int_{\gamma}^{\infty} e^{-s t}\left|t_{0}^{n-1}{ }_{0}^{C} D_{t}^{\alpha} f(t)\right| d t \leq s \int_{\gamma}^{\infty} K e^{\beta t-s t} d t \\
& \quad=\lim _{c \longrightarrow \infty}-\left.s K \frac{e^{-t(s-\beta)}}{s-\beta}\right|_{\gamma} ^{c}=\frac{s K}{s-\beta} e^{-\gamma(s-\beta)} .
\end{aligned}
$$

As a result, the ARA transform of ${ }_{0}^{C} D_{t}^{\alpha} f(t)$ exists.

Theorem 13 (ARA transform of Liouville-Caputo derivative for $n=1)$. If the $\alpha^{\text {th }}$ order of Liouville-Caputo derivative ${ }_{0}^{C}$ $D_{t}^{\alpha} f(t)$ of the function $f(t)$ is piecewise continuous in every finite interval and fulfils

$$
\left|t^{n-1}{ }_{0}^{C} D_{t}^{\alpha} f(t)\right| \leq K e^{\beta t}
$$

where $m-1<\alpha \leq m$, then ARA transform of it for $n=1$ is computed as

$$
G_{1}\left[{ }_{0}^{C} D_{t}^{\alpha} f(t)\right](s)=s^{\alpha} G_{1}[f(t)](s)-\sum_{k=0}^{m-1} f^{(k)}(0) s^{\alpha-k}
$$

for all $s>\beta$.

Proof. The ARA transform of Liouville-Caputo derivative for $n=1$ can be written as

$$
G_{1}\left[{ }_{0}^{C} D_{t}^{\alpha} f(t)\right](s)=G_{1}\left[{ }_{0}^{R L} I_{t}^{m-\alpha} f^{(m)}(t)\right](s) .
$$

Taking $f^{(m)}(t)=g(t)$ and using Theorem 11 lead to

$$
G_{1}\left[\begin{array}{l}
R L \\
0
\end{array} I_{t}^{m-\alpha} g(t)\right](s)=\frac{1}{s^{m-\alpha}} G_{1}[g(t)](s)
$$

Replacing $g(t)$ by $f^{(m)}(t)$ and utilizing integration by parts lead us to the following result

$$
\frac{1}{s^{m-\alpha}} G_{1}\left[f^{(m)}(t)\right](s)=s^{\alpha} G_{1}[f(t)](s)-\sum_{k=0}^{m-1} f^{(k)}(0) s^{\alpha-k} .
$$

\section{Main Results}

In this section, the implementation of IAM for nonlinear fractional partial differential equation with initial conditions is presented. Let us consider the following nonlinear fractional initial value problem:

$$
\begin{gathered}
{ }_{0}^{C} D_{t}^{\alpha}(u(x, y, t))+R u(x, y, t)+N u(x, y, t) \\
=g(x, y, t), m-1<\alpha \leq m, m \in \mathbb{N}
\end{gathered}
$$

$\frac{\partial^{k}}{\partial t^{k}} u(x, y, 0)=h_{k}(x, y), k=0,1,2, \cdots, m-1$,

where ${ }_{0}^{C} D_{t}^{\alpha}(u(x, y, t)), R, N$, and $g(x, y, t)$ represent fractional derivative, the linear equation operator, the general nonlinear differential operator, and the source term, respectively. In order to apply ARA transform to nonlinear fractional initial value problem, $u(x, y, t)$ must be $m$ times continuously differentiable function with respect to variable $t$ based on Definition 5. In other words, $u(x, y, t)$ must belong to the Banach space $W_{2}^{m}[0, T]$ where it is defined as

$$
W_{2}^{m}[0, T]=\left\{u=u(. . ., t): u, u^{(1)}, \cdots, u^{(m-1)} \in A C[0, T]\right\} .
$$

Without loss of generality taking $u=u(x, y, t)$. Making use of the ARA transform on equation (29), we obtain

$$
G_{1}\left[{ }_{0}^{C} D_{t}^{\alpha} u\right](s)+G_{1}[R u+N u](s)=G_{1}[g(x, y, t)](s) .
$$

Taking the property of the ARA transform into account leads to

$$
\begin{gathered}
s^{\alpha} G_{1}[u](s)-\sum_{k=0}^{m-1} \frac{\partial^{k}}{\partial t^{k}} u(\mathrm{x}, y, 0) s^{\alpha-k}+G_{1}[R u+N u](s) \\
=G_{1}[g(x, y, t)](s), \\
G_{1}[u](s)=\frac{1}{s^{\alpha}} \sum_{k=0}^{m-1} \frac{\partial^{k}}{\partial t^{k}} u(x, y, 0) s^{\alpha-k}-\frac{1}{s^{\alpha}} G_{1}[R u+N u](s) \\
+\frac{1}{s^{\alpha}} G_{1}[g(x, y, t)](s) .
\end{gathered}
$$

Employing inverse ARA transform gives

$$
\begin{aligned}
u= & G_{1}^{-1}\left[\frac{1}{s^{\alpha}}\left[\sum_{k=0}^{m-1} \frac{\partial^{k}}{\partial t^{k}} u(x, y, 0) s^{\alpha-k}+G_{1}[g(x, y, t)](s)\right]\right] \\
& -G_{1}^{-1}\left[\frac{1}{s^{\alpha}}\left[G_{1}[R u+N u](s)\right]\right] .
\end{aligned}
$$

Now, the application of the iterative method produces

$$
u=\sum_{i=0}^{\infty} u_{i}
$$

Linearity of the operator $R$ leads to

$$
R\left(\sum_{i=0}^{\infty} u_{i}\right)=\sum_{i=0}^{\infty} R\left(u_{i}\right)
$$


and decomposition of the nonlinear operator $N$ can be written as

$$
N\left(\sum_{i=0}^{\infty} u_{i}\right)=N\left(u_{0}\right)+\sum_{i=0}^{\infty}\left\{N\left(\sum_{k=0}^{i} u_{k}\right)-N\left(\sum_{k=0}^{i-1} u_{k}\right)\right\}
$$

Plugging (35), (36), and (37) in (34) allows us to have

$$
\begin{aligned}
\sum_{i=0}^{\infty} u_{i}= & G_{1}^{-1}\left[\frac{1}{s^{\alpha}}\left[\sum_{k=0}^{m-1} \frac{\partial^{k}}{\partial t^{k}} u(x, y, 0) s^{\alpha-k}+G_{1}[g(x, y, t)](s)\right]\right] \\
& -G_{1}^{-1}\left[\frac { 1 } { s ^ { \alpha } } \left[G _ { 1 } \left[R\left(u_{i}\right)+N\left(u_{0}\right)\right.\right.\right. \\
& \left.\left.\left.+\sum_{i=1}^{\infty}\left\{N\left(\sum_{k=0}^{i} u_{k}\right)-N\left(\sum_{k=0}^{i-1} u_{k}\right)\right\}\right](s)\right]\right] .
\end{aligned}
$$

The recurrence relation is obtained as follows:

$$
\begin{aligned}
u_{0}=G_{1}^{-1}\left[\frac{1}{s^{\alpha}}\left[\sum_{k=0}^{m-1} \frac{\partial^{k}}{\partial t^{k}} u(x, y, 0) s^{\alpha-k}+G_{1}[g(x, y, t)](s)\right]\right] \\
u_{1}=-G_{1}^{-1}\left[\frac{1}{s^{\alpha}}\left[G_{1}\left[R\left(u_{0}\right)+N\left(u_{0}\right)\right](s)\right]\right] \\
u_{m+1}=G_{1}^{-1}\left[\frac { 1 } { s ^ { \alpha } } \left[G _ { 1 } \left[R\left(u_{m}\right)-\left\{N\left(\sum_{k=0}^{m} u_{k}\right)\right.\right.\right.\right. \\
\left.\left.\left.-N\left(\sum_{k=0}^{m-1} u_{k}\right)\right\}\right](s)\right], m \geq 1
\end{aligned}
$$

Finally, the approximate solution of $m$-term is constructed as

$$
u(x, y, t) \cong u_{0}+u_{1}+u_{2}+\cdots+u_{m}, m=1,2, \cdots
$$

Theorem 14. In the Banach space $W_{2}^{m}[0, T]$, the solution of nonlinear fractional differential equation (29) in series form is convergent if the following inequality satisfies for some constant $\gamma, 0<\gamma<1$

$$
\begin{aligned}
\| G_{1}^{-1}\left[\frac{1}{s^{\alpha}}\left[G_{1}\left[R\left(u_{m}\right)-\left\{N\left(\sum_{k=0}^{m} u_{k}\right)-N\left(\sum_{k=0}^{m-1} u_{k}\right)\right\}\right](s)\right] \|\right. \\
\leq \gamma \| G_{1}^{-1}\left[\frac { 1 } { s ^ { \alpha } } \left[G _ { 1 } \left[R\left(u_{m-1}\right)-\left\{N\left(\sum_{k=0}^{m-1} u_{k}\right)\right.\right.\right.\right. \\
\left.\left.\left.\left.-N\left(\sum_{k=0}^{m-2} u_{k}\right)\right\}\right](s)\right]\right] \|, \forall m \in \mathbb{N},
\end{aligned}
$$

or $\left\|u_{m+1}\right\| \leq \gamma\left\|u_{m}\right\|$
Proof.

$$
S_{m}(x, y, t)=u_{1}+u_{2}+u_{3}+\cdots+u_{m}
$$

represent the sequences of partial sums for $m \geq 1$. In order to prove that the series solution of nonlinear fractional differential equation (29) is convergent, it is enough to show that $\left\{S_{m}\right\}_{m \geq 1}$ is a Cauchy sequence in a given Banach space $B$. For this aim, we take

$$
\begin{aligned}
& \left\|S_{m+1}-S_{m}\right\| \\
& \quad\left\|G_{1}^{-1}\left[\frac{1}{s^{\alpha}}\left[G_{1}\left[R\left(u_{m}\right)-\left\{N\left(\sum_{k=0}^{m} u_{k}\right)-N\left(\sum_{k=0}^{m-1} u_{k}\right)\right\}\right](s)\right]\right]\right\| \\
& \quad \leq \gamma\left\|G_{1}^{-1}\left[\frac{1}{s^{\alpha}}\left[G_{1}\left[R\left(u_{m-1}\right)-\left\{N\left(\sum_{k=0}^{m-1} u_{k}\right)-N\left(\sum_{k=0}^{m-2} u_{k}\right)\right\}\right](s)\right]\right]\right\| \\
& \quad \leq \gamma^{2}\left\|G_{1}^{-1}\left[\frac{1}{s^{\alpha}}\left[G_{1}\left[R\left(u_{m-2}\right)-\left\{N\left(\sum_{k=0}^{m-2} u_{k}\right)-N\left(\sum_{k=0}^{m-3} u_{k}\right)\right\}\right](s)\right]\right]\right\| \\
& \quad \leq \cdots \leq \gamma^{n+1}\left\|G_{1}^{-1}\left[\frac{1}{s^{\alpha}}\left[\sum_{k=0}^{m-1} \frac{\partial^{k}}{\partial t^{k}} u(x, y, 0) s^{\alpha-k}+G_{1}[g(x, y, t)](s)\right]\right]\right\| .
\end{aligned}
$$

For every $n, m \in \mathbb{N}, n \geq m$, utilizing (43) and triangle inequality successively, we get

$$
\begin{aligned}
\left\|S_{n}-S_{m}\right\|= & \left\|S_{m+1}-S_{m}+S_{m+2}-S_{m+1}+\cdots+S_{n}-S_{n-1}\right\| \\
\leq & \gamma^{m+1}\left(\frac{1-\gamma^{n-m}}{1-\gamma}\right) \| G_{1}^{-1}\left[\frac { 1 } { s ^ { \alpha } } \left[\sum_{k=0}^{m-1} \frac{\partial^{k}}{\partial t^{k}} u(x, y, 0) s^{\alpha-k}\right.\right. \\
& \left.\left.+G_{1}[g(x, y, t)](s)\right]\right] \|
\end{aligned}
$$

The assumption $0<\gamma<1$ implies that $1-\gamma^{n-m} \leq 1$ which yields

$$
\begin{aligned}
\left\|S_{n}-S_{m}\right\| \leq & \frac{\gamma^{m+1}}{1-\gamma} \| G_{1}^{-1}\left[\frac { 1 } { s ^ { \alpha } } \left[\sum_{k=0}^{m-1} \frac{\partial^{k}}{\partial t^{k}} u(x, y, 0) s^{\alpha-k}\right.\right. \\
& \left.\left.+G_{1}[g(x, y, t)](s)\right]\right] \|
\end{aligned}
$$

Boundedness of $G_{1}^{-1}\left[1 / s^{\alpha}\left[\sum_{k=0}^{m-1}\left(\partial^{k} / \partial t^{k}\right) u(x, y, 0) s^{\alpha-k}+\right.\right.$ $\left.\left.G_{1}[g(x, y, t)](s)\right]\right]$ implies the following:

$$
\lim _{n, m \longrightarrow \infty}\left\|S_{n}-S_{m}\right\|=0
$$

This result implies that the sequence $\left\{S_{m}\right\}_{m \geq 1}$ is a Cauchy sequence in Banach space $B$. As a result, the series solution (35) is convergent. $\square$ 


\section{Illustrative Example}

As illustrative examples, nonlinear fractional initial value problems of biological population models are presented in this section to show how IAM is implemented.

Example 1. Consider the following generalized timefractional biological population model:

$$
{ }_{0}^{C} D_{t}^{\alpha} u=\frac{\partial^{2}}{\partial x^{2}} u^{2}+\frac{\partial^{2}}{\partial y^{2}} u^{2}+h u
$$

with the initial condition

$$
u(x, y, 0)=\sqrt{x y}
$$

where $u=u(x, y, t), 0<\alpha \leq 1,(x, y) \in \mathbb{R}^{2}, t>0$.

Employing the ARA transform on equation (49) with the initial condition (50) produces the following:

$$
G_{1}[u](s)=\sqrt{x y}+\frac{1}{s^{\alpha}} G_{1}\left[\frac{\partial^{2}}{\partial x^{2}} u^{2}+\frac{\partial^{2}}{\partial y^{2}} u^{2}+h u\right](s) .
$$
to

Applying inverse ARA transform to equation (51) leads

$$
u=\sqrt{x y}+G_{1}^{-1}\left[\frac{1}{s^{\alpha}}\left[G_{1}\left[\frac{\partial^{2}}{\partial x^{2}} u^{2}+\frac{\partial^{2}}{\partial y^{2}} u^{2}+h u\right](s)\right]\right] .
$$

Substituting (35)-(37) into (52) and utilizing (39), the components of the solution are established as follows:

$$
\begin{aligned}
u_{0}= & u(x, y, 0)=\sqrt{x y}, \\
u_{1}= & G_{1}^{-1}\left[\frac{1}{s^{\alpha}}\left[G_{1}\left[\frac{\partial^{2}}{\partial x^{2}} u_{0}^{2}+\frac{\partial^{2}}{\partial y^{2}} u_{0}^{2}+h u_{0}\right](s)\right]\right] \\
= & G_{1}^{-1}\left[\frac{1}{s^{\alpha}}\left[G_{1}[h \sqrt{x y}](s)\right]\right]=h \frac{t^{\alpha}}{\Gamma(\alpha+1)} \sqrt{x y}, \\
u_{2}= & G_{1}^{-1}\left[\frac { 1 } { s ^ { \alpha } } \left[G _ { 1 } \left[\frac{\partial^{2}}{\partial x^{2}}\left(u_{0}+u_{1}\right)^{2}+\frac{\partial^{2}}{\partial y^{2}}\left(u_{0}+u_{1}\right)^{2}\right.\right.\right. \\
& \left.\left.\left.+h\left(u_{0}+u_{1}\right)\right](s)\right]\right]-G_{1}^{-1}\left[\frac { 1 } { s ^ { \alpha } } \left[G _ { 1 } \left[\frac{\partial^{2}}{\partial x^{2}} u_{0}^{2}\right.\right.\right. \\
& \left.\left.\left.+\frac{\partial^{2}}{\partial y^{2}} u_{0}^{2}+h u_{0}\right](s)\right]\right] \\
= & G_{1}^{-1}\left[\frac{1}{s^{\alpha}}\left[G_{1}\left[\left(h+h^{2} \frac{t^{\alpha}}{\Gamma(\alpha+1)}\right) \sqrt{x y}\right](s)\right]\right] \\
& -h \frac{t^{\alpha}}{\Gamma(\alpha+1)} \sqrt{x y}=h^{2} \frac{t^{2 \alpha}}{\Gamma(2 \alpha+1)} \sqrt{x y},
\end{aligned}
$$

$$
\begin{aligned}
u_{3}= & G_{1}^{-1}\left[\frac { 1 } { s ^ { \alpha } } \left[G _ { 1 } \left[\frac{\partial^{2}}{\partial x^{2}}\left(u_{0}+u_{1}+u_{2}\right)^{2}+\frac{\partial^{2}}{\partial y^{2}}\left(u_{0}+u_{1}+u_{2}\right)^{2}\right.\right.\right. \\
& \left.\left.\left.+h\left(u_{0}+u_{1}+u_{2}\right)\right](s)\right]\right]-G_{1}^{-1}\left[\frac { 1 } { s ^ { \alpha } } \left[G _ { 1 } \left[\frac{\partial^{2}}{\partial x^{2}}\left(u_{0}+u_{1}\right)^{2}\right.\right.\right. \\
& \left.\left.\left.+\frac{\partial^{2}}{\partial y^{2}}\left(u_{0}+u_{1}\right)^{2}+h\left(u_{0}+u_{1}\right)\right](s)\right]\right] \\
= & G_{1}^{-1}\left[\frac{1}{s^{\alpha}}\left[G_{1}\left[\left(h+h^{2} \frac{t^{\alpha}}{\Gamma(\alpha+1)}+h^{3} \frac{t^{2 \alpha}}{\Gamma(2 \alpha+1)}\right) \sqrt{x y}\right](s)\right]\right] \\
& -h^{2} \frac{t^{2 \alpha}}{\Gamma(2 \alpha+1)} \sqrt{x y}-h \frac{t^{\alpha}}{\Gamma(\alpha+1)} \sqrt{x y}=\frac{t^{3 \alpha}}{\Gamma(3 \alpha+1)} \sqrt{x y}
\end{aligned}
$$

Finally, the numerical solution of the problem is constructed as

$$
\begin{aligned}
u \cong & u_{0}+u_{1}+u_{2}+\cdots+u_{m}, \\
u \cong & \left(1+h \frac{t^{\alpha}}{\Gamma(\alpha+1)}+h^{2} \frac{t^{2 \alpha}}{\Gamma(2 \alpha+1)}\right. \\
& \left.+h^{3} \frac{t^{3 \alpha}}{\Gamma(3 \alpha+1)}+\cdots+h^{m} \frac{t^{m \alpha}}{\Gamma(m \alpha+1)}\right) \sqrt{x y} .
\end{aligned}
$$

As $m$ tends to infinity, the numerical solution converges to following analytical solution

$$
u(x, y, t)=E_{\alpha, 1}\left(h t^{\alpha}\right) \sqrt{x y} .
$$

The exact solution which is computed by taking $\alpha=1$ can be written as [18]:

$$
u(x, y, t)=\exp (h t) \sqrt{x y} .
$$

Example 2. Let us take the following generalized timefractional biological population model into consideration:

$$
{ }_{0}^{C} D_{t}^{\alpha} u=\frac{\partial^{2}}{\partial x^{2}} u^{2}+\frac{\partial^{2}}{\partial y^{2}} u^{2}+u
$$

with the initial condition

$$
u(x, y, 0)=\sqrt{\sin (x) \sin h(y)},
$$

where $u=u(x, y, t), 0<\alpha \leq 1,(x, y) \in \mathbb{R}^{2}, t>0$.

Utilizing the ARA transform on equation (57) with the initial condition (58) produces the following:

$$
G_{1}[u](s)=\sqrt{\sin (x) \sin h(y)}+\frac{1}{s^{\alpha}} G_{1}\left[\frac{\partial^{2}}{\partial x^{2}} u^{2}+\frac{\partial^{2}}{\partial y^{2}} u^{2}+u\right](s) .
$$


TABLE 1: The absolute errors between the approximate solutions $u_{7}$ and the exact solution for Examples 1, 2, and 3 at $\alpha=1$.

\begin{tabular}{lccc}
\hline & Example 1 & Example 2 & $\begin{array}{c}\text { Example 3 } \\
h=1, r=2\end{array}$ \\
\hline$(0.1,0.1,0.1)$ & $2.509104035652854 \times 10^{-14}$ & $2.50771625687207 \times 10^{-14}$ & $2.771116669464391 \times 10^{-13}$ \\
$(0.2,0.2,0.2)$ & $1.298636198576730 \times 10^{-11}$ & $1.298622320788923 \times 10^{-11}$ & $7.930767154107343 \times 10^{-11}$ \\
$(0.3,0.3,0.3)$ & $5.04943808987690 \times 10^{-10}$ & $5.049211049268365 \times 10^{-10}$ & $2.27200946945061 \times 10^{-9}$ \\
$(0.4,0.4,0.4)$ & $6.802539864736446 \times 10^{-9}$ & $6.801572194348182 \times 10^{-9}$ & $2.537049281770010 \times 10^{-8}$ \\
$(0.5,0.5,0.5)$ & $5.127268321025724 \times 10^{-8}$ & $5.125487789747751 \times 10^{-8}$ & $1.690687270183844 \times 10^{-7}$ \\
\hline
\end{tabular}
to

Applying inverse ARA transform to equation (59) leads

$u=\sqrt{\sin (x) \sin h(y)}+G_{1}^{-1}\left[\frac{1}{s^{\alpha}}\left[G_{1}\left[\frac{\partial^{2}}{\partial x^{2}} u^{2}+\frac{\partial^{2}}{\partial y^{2}} u^{2}+u\right](s)\right]\right]$

Substituting (35)-(37) into (60) and utilizing (39), the components of the solution are established as follows:

$$
\begin{aligned}
& u_{0}=u(x, y, 0)=\sqrt{\sin (x) \sin h(y)}, \\
& u_{1}=\frac{t^{\alpha}}{\Gamma(\alpha+1)} \sqrt{\sin (x) \sin h(y)}, \\
& u_{2}=\frac{t^{2 \alpha}}{\Gamma(2 \alpha+1)} \sqrt{\sin (x) \sin h(y)}, \\
& u_{3}=\frac{t^{3 \alpha}}{\Gamma(3 \alpha+1)} \sqrt{\sin (x) \sin h(y)},
\end{aligned}
$$

Finally, the numerical solution of the problem is constructed as

$$
\begin{aligned}
u \cong & u_{0}+u_{1}+u_{2}+\cdots+u_{m}, \\
u \cong & \left(1+\frac{t^{\alpha}}{\Gamma(\alpha+1)}+\frac{t^{2 \alpha}}{\Gamma(2 \alpha+1)}+\frac{t^{3 \alpha}}{\Gamma(3 \alpha+1)}\right. \\
& \left.+\cdots+\frac{t^{m \alpha}}{\Gamma(m \alpha+1)}\right) \sqrt{\sin (x) \sin h(y)} .
\end{aligned}
$$

As $m$ goes to infinity, the numerical solution converges to following analytical solution

$$
u(x, y, t)=E_{\alpha, 1}\left(t^{\alpha}\right) \sqrt{\sin (x) \sin h(y)} .
$$

The exact solution which is computed by taking $\alpha=1$ can be written as [19]:

$$
u(x, y, t)=\exp (t) \sqrt{\sin (x) \sin h(y)} .
$$

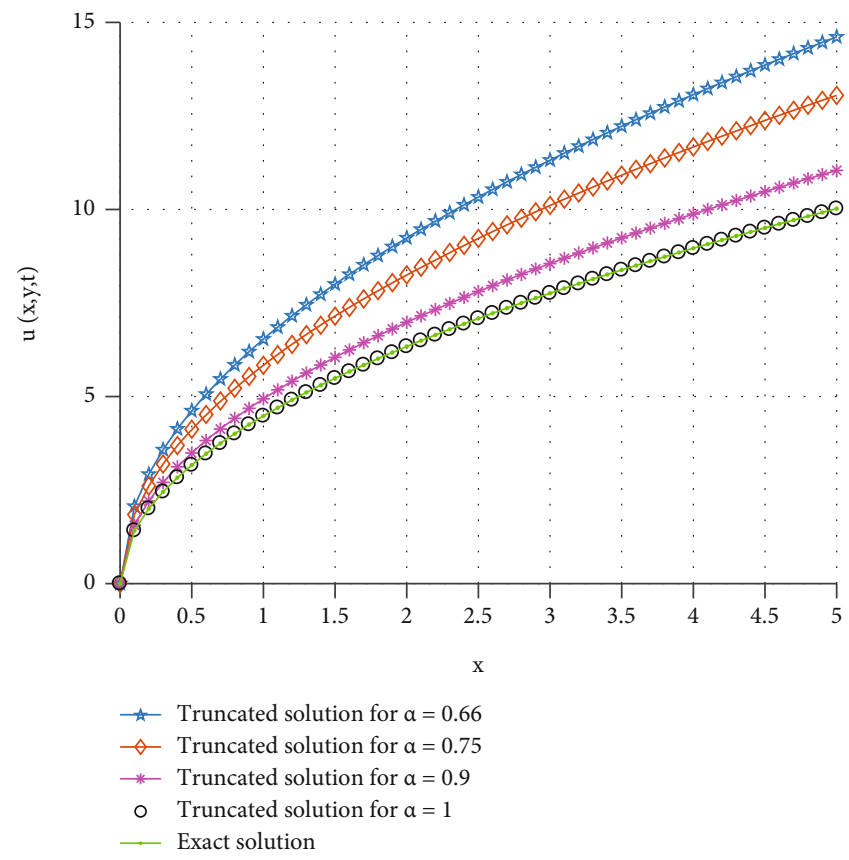

FIgURE 1: The 2D graphs of exact and approximate solutions $u_{7}$ by IAM for various of $\alpha$ for Example 1 at $t=1.5$ and $h=y=1$.

Example 3. Consider the following generalized timefractional biological population model:

$$
{ }_{0}^{C} D_{t}^{\alpha} u=\frac{\partial^{2}}{\partial x^{2}} u^{2}+\frac{\partial^{2}}{\partial y^{2}} u^{2}+h u(1-r u)
$$

with the initial condition

$$
u(x, y, 0)=\exp \left(\sqrt{\frac{h r}{8}}(x+y)\right)
$$

where $u=u(x, y, t), 0<\alpha \leq 1,(x, y) \in \mathbb{R}^{2}, t>0$.

Employing the ARA transform on equation (65) with the initial condition (66) produces the following:

$$
\begin{aligned}
G_{1}[u](s)= & \exp \left(\sqrt{\frac{h r}{8}}(x+y)\right)+\frac{1}{s^{\alpha}} G_{1}\left[\frac{\partial^{2}}{\partial x^{2}} u^{2}\right. \\
& \left.+\frac{\partial^{2}}{\partial y^{2}} u^{2}+h u(1-r u)\right](s) .
\end{aligned}
$$




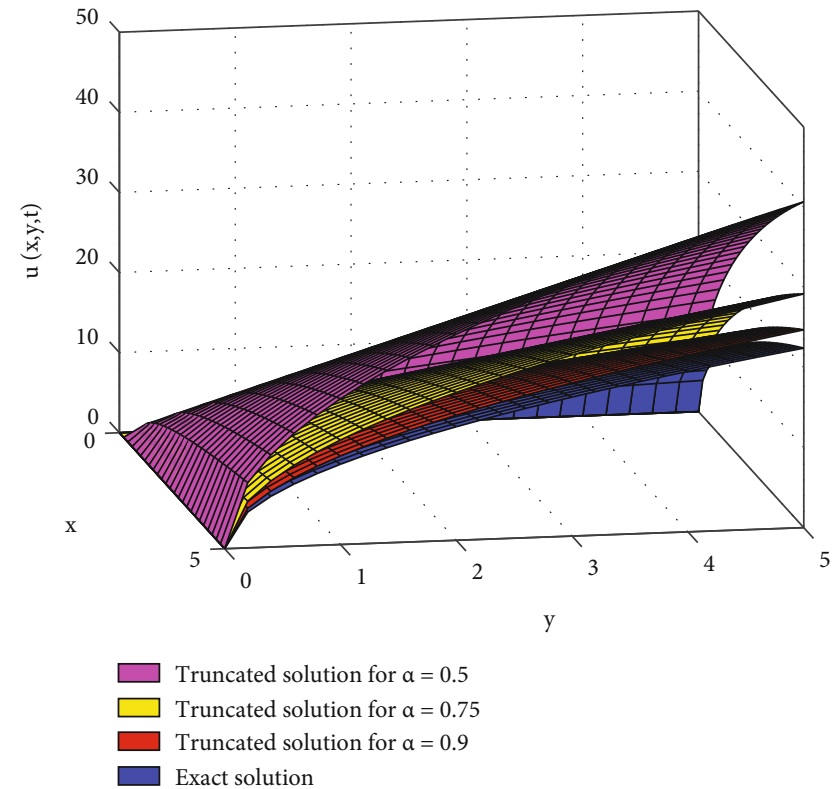

Figure 2: 3D graphs of exact and approximate solutions $u_{7}$ by IAM for various values of $\alpha$ for Example 1 at $t=1.5$ and $h=1$.

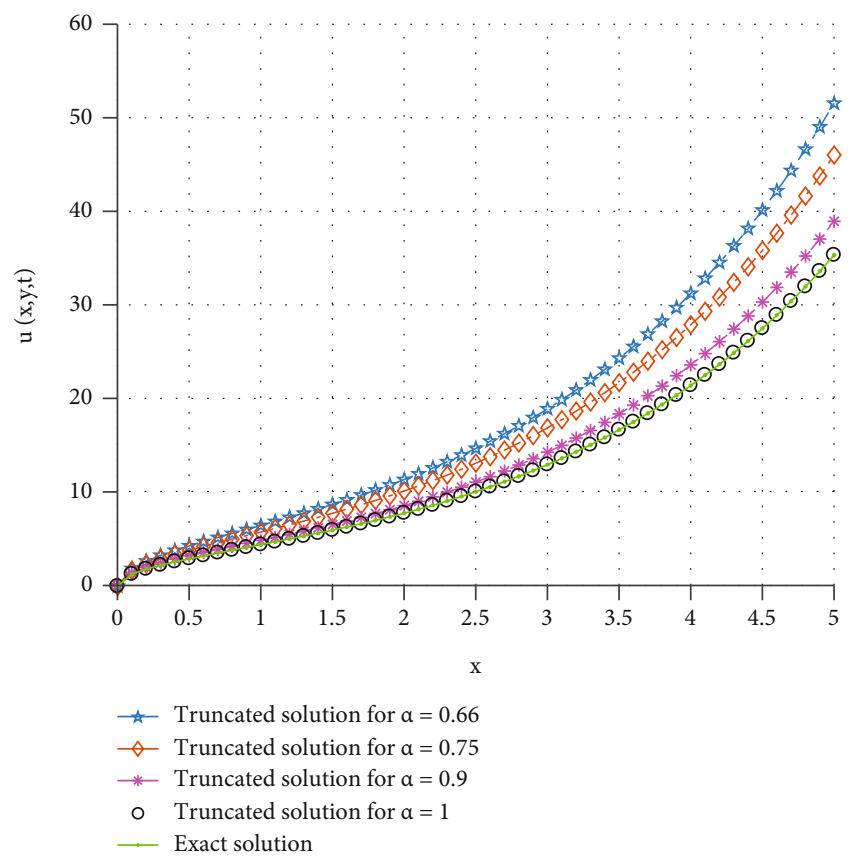

FIGURE 3: The 2D graphs of exact and approximate solutions $u_{7}$ by IAM for various of $\alpha$ for Example 2 at $t=1.5$ and $x=1$.

Applying inverse ARA transform to equation (67) leads to

$$
\begin{aligned}
u= & \exp \left(\sqrt{\frac{h r}{8}}(x+y)\right)+G_{1}^{-1}\left[\frac { 1 } { s ^ { \alpha } } \left[G _ { 1 } \left[\frac{\partial^{2}}{\partial x^{2}} u^{2}\right.\right.\right. \\
& \left.\left.\left.+\frac{\partial^{2}}{\partial y^{2}} u^{2}+h u(1-r u)\right](s)\right]\right]
\end{aligned}
$$

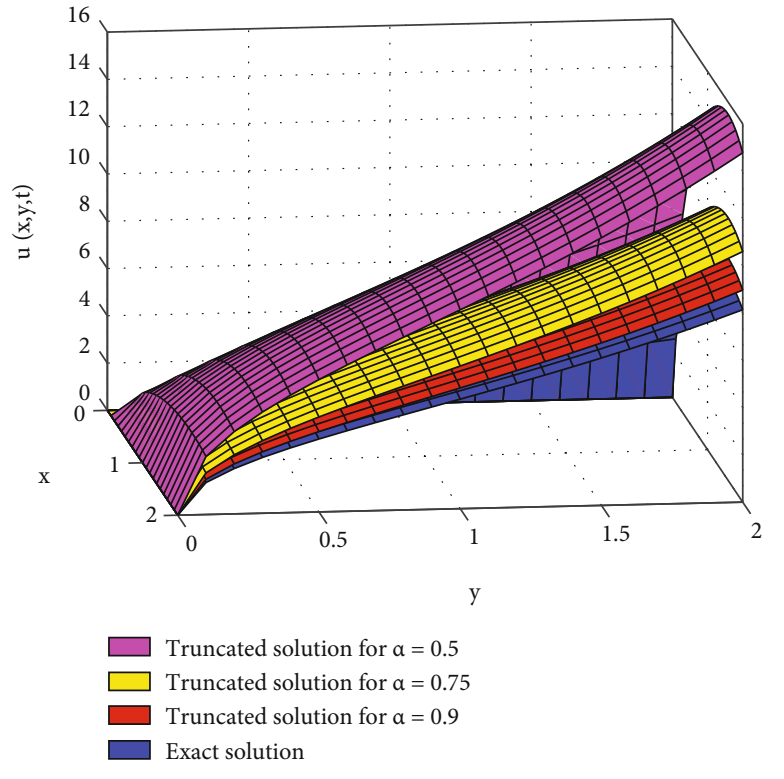

FIGURE 4: 3D graphs of exact and approximate solutions $u_{7}$ by IAM for various values of $\alpha$ for Example 2 at $t=1.5$.

Substituting (35)-(37) into (68) and utilizing (39), the components of the solution are established as follows:

$$
\begin{aligned}
& u_{0}=u(x, y, 0)=\exp \left(\sqrt{\frac{h r}{8}}(x+y)\right), \\
& u_{1}=h \frac{t^{\alpha}}{\Gamma(\alpha+1)} \exp \left(\sqrt{\frac{h r}{8}}(x+y)\right), \\
& u_{2}=h^{2} \frac{t^{2 \alpha}}{\Gamma(2 \alpha+1)} \exp \left(\sqrt{\frac{h r}{8}}(x+y)\right), \\
& u_{3}=h^{3} \frac{t^{3 \alpha}}{\Gamma(3 \alpha+1)} \exp \left(\sqrt{\frac{h r}{8}}(x+y)\right),
\end{aligned}
$$

Finally, the numerical solution of the problem is constructed as

$$
\begin{gathered}
u \cong u_{0}+u_{1}+u_{2}+\cdots+u_{m}, \\
u \cong\left(1+h \frac{t^{\alpha}}{\Gamma(\alpha+1)}+h^{2} \frac{t^{2 \alpha}}{\Gamma(2 \alpha+1)}+h^{3} \frac{t^{3 \alpha}}{\Gamma(3 \alpha+1)}\right. \\
\left.+\cdots+h^{m} \frac{t^{m \alpha}}{\Gamma(m \alpha+1)}\right) \exp \left(\sqrt{\frac{h r}{8}}(x+y)\right) .
\end{gathered}
$$

As $m$ tends to infinity, the numerical solution converges to following analytical solution

$$
u(x, y, t)=E_{\alpha, 1}\left(h t^{\alpha}\right) \exp \left(\sqrt{\frac{h r}{8}}(x+y)\right) .
$$

The exact solution which is computed by taking $\alpha=1$ 


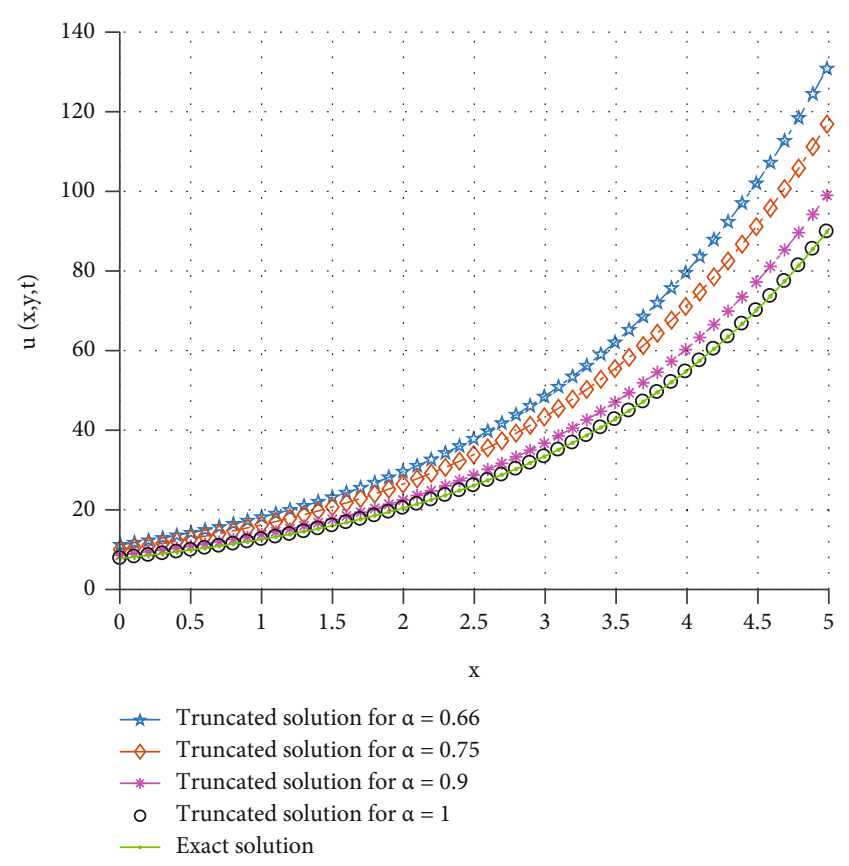

FIgURE 5: The 2D graphs of exact and approximate solutions $u_{7}$ by IAM for various of $\alpha$ for Example 3 at $t=1.5, r=2$, and $y=h=1$.

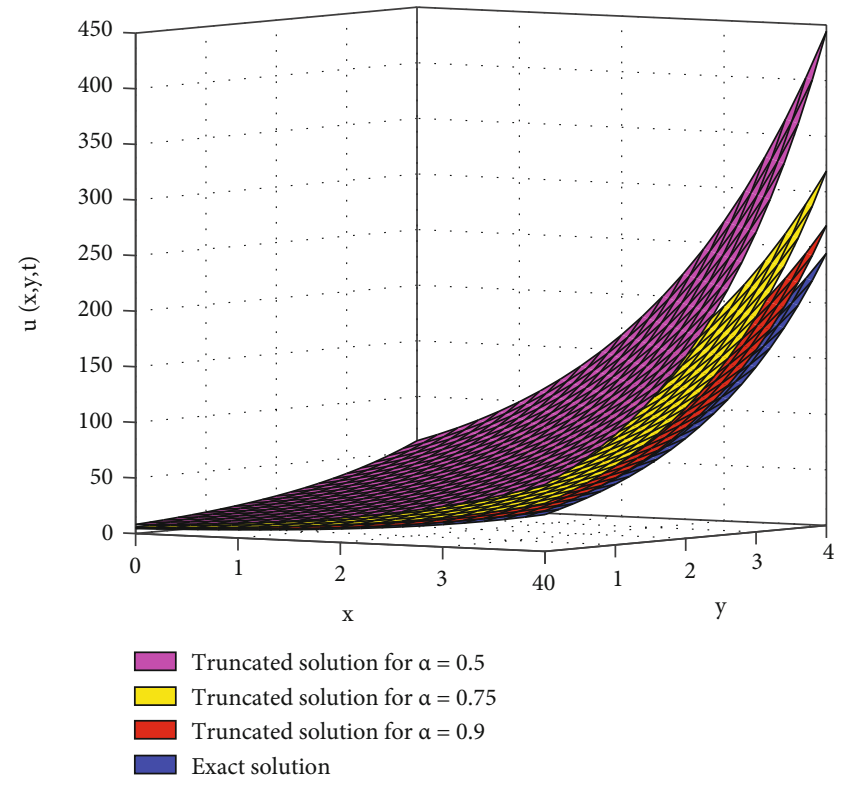

FIGURE 6: $3 \mathrm{D}$ graphs of exact and approximate solutions $u_{7}$ by IAM for various values of $\alpha$ for Example 3 at $t=1.5, r=2$, and $h=1$.

can be written as [20]:

$$
u(x, y, t)=\exp \left(\sqrt{\frac{h r}{8}}(x+y)+h t\right)
$$

In Table 1, absolute errors between the exact solution and the approximate solutions $u_{7}$ at $\alpha=1$ for Examples 1, 2 , and 3 are presented. Figures $1-6$ and outcomes verify that when the order of the fractional derivative $\alpha$ increases to 1 , the truncated solutions obtained by IAM continuously get closer to the exact solutions. Notice that the outcomes are compatible with 2D and 3D graphs of exact and approximate solutions $u_{7}$. Furthermore, we concluded that increasing the number of terms in approximate solutions implies the improvement of accuracy of approximate solutions.

\section{Conclusion}

In this study, nonlinear fractional initial value problem is investigated by a new numerical method IAM which is a combination of the ARA transform and iterative methods. The implementation and convergence analysis of IAM shows how effective and accurate this method is. As an application of this model, Liouville-Caputo time-fractional biological population problems are taken into consideration. The outcomes of these examples also support the effectiveness and accuracy of the method.

In the future works, the utilization of ARA transform with other numerical methods is taken into account to analyze substantial nonlinear mathematical problems.

\section{Data Availability}

No data were used to support this study.

\section{Conflicts of Interest}

The authors declare that there is no conflict of interest regarding the publication of this paper.

\section{Authors' Contributions}

All authors contributed equally to this work.

\section{References}

[1] M. M. Khader and K. M. Saad, "A numerical study using Chebyshev collocation method for a problem of biological invasion: fractional Fisher equation," International Journal of Biomathematics, vol. 11, article 1850099, 2018.

[2] K. M. Saad, S. Deniz, and D. Baleanu, "On a new modified fractional analysis of Nagumo equation," International Journal of Biomathematics, vol. 12, article 1950034, 2019.

[3] K. M. Saad, M. M. Khader, J. F. Gómez-Aguilar, and D. Baleanu, "Numerical solutions of the fractional Fisher's type equations with Atangana-Baleanu fractional derivative by using spectral collocation methods," Chaos, vol. 29, article 023116, 2019.

[4] K. M. Saad, D. Baleanu, and A. Atangana, "New fractional derivatives applied to the Korteweg-de Vries and Kortewegde Vries-Burger's equations," Computational and Applied Mathematics, vol. 37, pp. 5203-5216, 2018.

[5] K. M. Saad and A. L.-S. EH, "Comparative study of a cubic autocatalytic reaction via different analysis methods," Discrete \& Continuous Dynamical Systems-S, vol. 12, pp. 665-684, 2019.

[6] O. Acana, M. M. Al Qurashib, and D. Baleanu, "Reduced differential transform method for solving time and space local fractional partial differential equations," Journal of Nonlinear Sciences and Applications, vol. 10, pp. 5230-5238, 2017. 
[7] Z. Hammouch and T. Mekkaoui, "A domian decomposition method for solving a time-fractional Burger-Huxley's equation," Nonlinear Studies, vol. 19, pp. 489-496, 2012.

[8] A. M. A. el-Sayed, A. Elsaid, I. L. el-Kalla, and D. Hammad, “A homotopy perturbation technique for solving partial differential equations of fractional order in finite domains," Applied Mathematics and Computation, vol. 218, no. 17, pp. 83298340, 2012.

[9] V. Turut and N. Güzel, "On solving partial differential equations of fractional order by using the variational iteration method and multivariate Pad'e approximations," European Journal of Pure and Applied Mathematics, vol. 6, pp. 147171, 2013.

[10] S. Cetinkaya, A. Demir, and H. Kodal Sevindir, "Solution of space-time-fractional problem by Shehu variational iteration method," Advances in Mathematical Physics, vol. 2021, Article ID 5528928, 2021.

[11] A. Elsaid, "Homotopy analysis method for solving a class of fractional partial differential equations," Communications in Nonlinear Science and Numerical Simulation, vol. 16, pp. 3655-3664, 2011.

[12] I. Podlubny, Fractional Differential Equations, Academic Press, New York, 1999.

[13] A. Khalouta and A. Kadem, "Comparison of new iterative method and natural homotopy perturbation method for solving nonlinear time-fractional wave-like equations with variable coefficients," Nonlinear Dynamics and Systems Theory, vol. 19, pp. 160-169, 2019.

[14] R. Saadeh, A. Qazza, and A. Burqan, "A new integral transform: ARA transform and its properties and applications," Symmetry, vol. 12, p. 925, 2020.

[15] S. Bhalekar and V. Daftardar-Gejji, "Solving evolution equations using a new iterative method," Numerical Methods for Partial Differential Equations, vol. 26, no. 4, pp. 906-916, 2010.

[16] A. A. Kilbas, H. M. Srivastava, and J. J. Trujlllo, Theory and Applications of Fractional Differential Equations, Elsevier, 2006.

[17] A. K. Shukla and J. C. Prajapati, "On a generalization of Mittag-Leffler function and its properties," Journal of Mathematical Analysis and Applications, vol. 336, no. 2, pp. 797811, 2007.

[18] Y. Liu, Z. Li, and Y. Zhang, "Homotopy perturbation method to fractional biological population equation," Fractional Differential Calculus, vol. 1, pp. 117-124, 2011.

[19] P. Roul, "Application of homotopy perturbation method to perturbation biological population model," Applications and Applied Mathematics, vol. 5, pp. 272-281, 2010.

[20] A. A. M. Arafa, S. Z. Rida, and H. Mohamed, "Homotopy analysis method for solving biological population model," Communications in Theoretical Physics, vol. 56, pp. 797-800, 2011. 